\title{
Using robots to understand social behaviour
}

\author{
Sara Mitri ${ }^{1, *}$, Steffen Wischmann², Dario Floreano ${ }^{2}$ and Laurent Keller ${ }^{3}$ \\ ${ }^{1}$ Department of Zoology, University of Oxford, South Parks Road, Oxford OX1 3PS, U.K. \\ ${ }^{2}$ Laboratory of Intelligent Systems, Ecole Polytechnique Fédérale de Lausanne, EPFL, Station 11, 1015 Lausanne, Switzerland \\ ${ }^{3}$ Department of Ecology and Evolution, University of Lausanne, Biophore, 1015 Lausanne, Switzerland
}

\begin{abstract}
A major challenge in studying social behaviour stems from the need to disentangle the behaviour of each individual from the resulting collective. One way to overcome this problem is to construct a model of the behaviour of an individual, and observe whether combining many such individuals leads to the predicted outcome. This can be achieved by using robots. In this review we discuss the strengths and weaknesses of such an approach for studies of social behaviour. We find that robots - whether studied in groups of simulated or physical robots, or used to infiltrate and manipulate groups of living organisms - have important advantages over conventional individual-based models and have contributed greatly to the study of social behaviour. In particular, robots have increased our understanding of self-organization and the evolution of cooperative behaviour and communication. However, the resulting findings have not had the desired impact on the biological community. We suggest reasons for why this may be the case, and how the benefits of using robots can be maximized in future research on social behaviour.
\end{abstract}

Key words: robot, social behaviour, collective behaviour, individual-based model (IBM), simulation, model.

\section{CONTENTS}

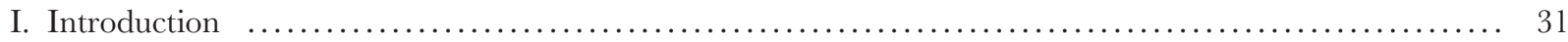

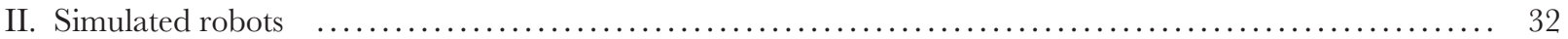

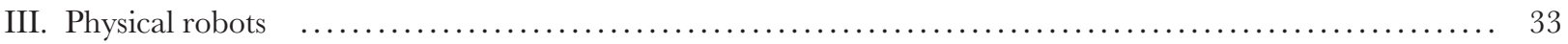

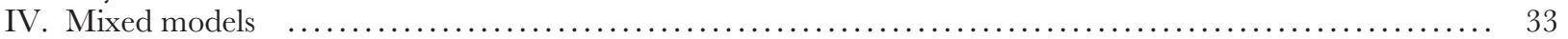

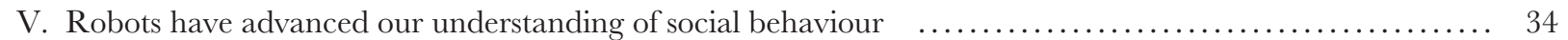

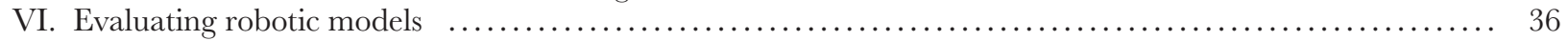

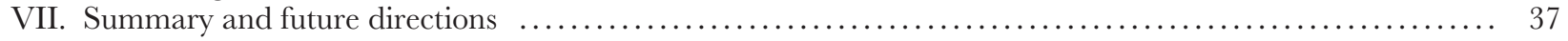

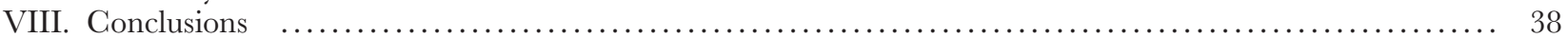

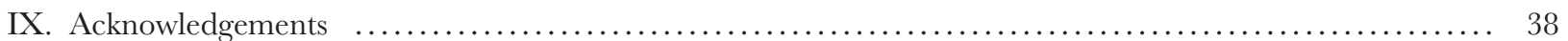

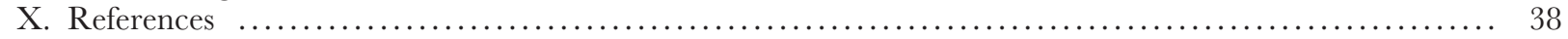

\section{INTRODUGTION}

Social behaviour, both in humans and other organisms, has for many years drawn the attention of researchers from a range of fields, as it poses interesting questions from mechanistic and evolutionary viewpoints. Many approaches have been used to study social behaviour. These approaches can be classified over a scale of 'situatedness', which we define as the extent to which individuals are embedded in an environment that can be sensed and modified by those individuals (Varela, Thompson \& Rosch, 1991; Clark, 1996). The situatedness spectrum ranges from abstract mathematical models at one end to field work in natural habitats at the other (Fig. 1).

At one extreme of the situatedness scale, observational or experimental studies performed in the field are useful in that they include the whole complexity of the organisms and their environment. However, while such studies allow one to infer correlations, they rarely permit the unambiguous demonstration of causations, for example in how the behaviour of an organism is affected by those of other individuals in the population. The realization of this limitation has led to an active field of experimental studies performed in the laboratory where it is easier to control variables of interest.

* Address for correspondence (E-mail: sara.mitri@a3.epfl.ch). 


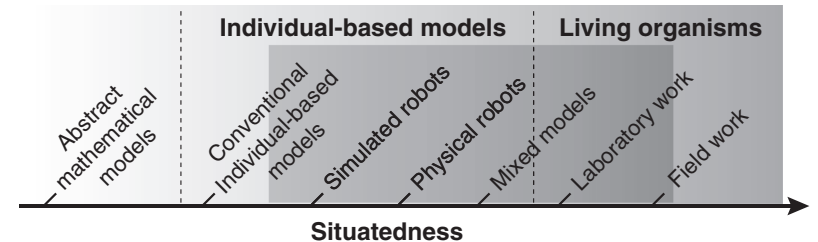

Fig. 1. Different approaches to studying social behaviour on a scale of situatedness, i.e. the extent to which individuals are embedded in an environment that they can sense and modify. The shaded box represents the robotic models covered herein.

While these studies have provided important insights into the social behaviour of organisms, an important limitation is that it is frequently difficult or impossible to manipulate the behaviour of individuals to investigate the response of other group members.

At the other end of the scale, abstract mathematical models allow one to boil down collective systems to their minimal components and explore the effects of what are considered to be key parameters on their dynamics. While abstract models can make powerful predictions, they generally model populations as a whole thereby neglecting or strongly simplifying the spatial environment, local interactions, lifecycle dynamics and phenotypic plasticity (DeAngelis \& Mooij, 2005). The realization of the importance of these factors has led to the development of individual-based models [IBMs, a term often used interchangeably with 'agent-based models' (Grimm \& Railsback, 2005)], where 'agents' are modelled individually. Such models allow one to consider individual differences such as age or size and their possible interactions (Judson, 1994; Grimm \& Railsback, 2005). These agent-based models have played an important role in explaining social behaviours, and have thus largely been accepted as part of the toolbox for modelling social systems from small groups to populations and ecosystems (DeAngelis \& Mooij, 2005). Agents in these IBMs are typically implemented using computer simulations.

More recently, researchers have resorted to using robots spanning different levels of situatedness, including simulated robots, physical robots, and 'mixed models' where physical robots and animals interact (Garnier, 2011; Krause, Winfield \& Deneubourg, 2011). The aim of this review is to discuss how the use of robots can complement experimental and theoretical studies on social behaviour.

Robots have been introduced as a means to study social behaviour relatively recently, and the approach has quickly gained momentum [see Garnier (2011) and Krause, Winfield \& Deneubourg (2011) for recent reviews]. We define a physical robot as 'a machine that is able to interact physically with its environment and perform some sequence of behaviours, either autonomously or by remote control' (Krause, Winfield \& Deneubourg, 2011). Essentially, robotic models of collective behaviour are IBMs in which individually programmed robots interact. As in conventional IBMs, all components of the individual robots are given, making it easier to understand their collective behaviour, compared to that of living organisms. However, the main advantage of using robots in a real physical environment over IBMs within a simulated environment is that fewer assumptions need to be made regarding the environmental properties (e.g. spatial constraints, perceptual noise, signal propagation). This is because the laws of physics are included 'for free' in robotic models. A direct consequence of this is that experiments using robots are more likely to lead to unexpected and interesting outcomes whenever some property of the physical world that would intuitively not have been included in an abstract environment has an important influence on the resulting collective behaviour. This assumes, however, that these physical properties and the resulting behaviour are not caused by artefacts that are specific to the robots and do not have parallels in the natural world.

So far, most of the studies involving robots have been conducted by computer scientists and engineers with the effect that much of the published work is unknown to biologists. It is therefore timely to review these studies, assess the extent to which they have contributed to our understanding of social behaviour, and outline the most promising directions for future research. Herein, we classify robotic models used to study social behaviour into three categories: $(i)$ simulated robots, where the physical environment and the robots are modelled in computer simulations, (ii) physical robots where experiments are conducted with real robots, and (iii) mixed models where physical robots interact with living organisms (Fig. 1).

\section{SIMULATED ROBOTS}

Simulated robots, here defined as computer simulations of physical robots and their environment, are at an intermediate level on the situatedness scale between conventional IBMs and experiments with physical robots (Fig. 1). While simulated robots may first appear to be equivalent to conventional IBMs, they differ critically in that they are designed to mimic physical robots and their environment, thus forcing the modeller to take constraints in perception, actuation, space and resources into account. Simulated robots have three characteristics: (i) they have an extended body (i.e. they occupy space in the world) rather than being a point; (ii) they gather information about the environment through sensors that are morphologically located and limited in range and accuracy rather than having an ideal perceptual system that can access global and perfect information; and (iii) they move in an extended space with finite distances and resources rather than in mathematical spaces which often have no boundaries and/or infinite resources (see e.g. Fig. 2A). All these factors can significantly affect the outcome of social behaviours that imply physical and perceptual interactions within a confined space. In addition, complete knowledge of the components and functioning of the modelled physical robot and its environment reduces the number of assumptions that need to be made when constructing the simulation, compared to a conventional 
IBM, which models living organisms that may not be fully understood.

The recent rise in computational power and simulation technology (Waldner, 2008) has led to the development of offthe-shelf robotic simulators that include models of a number of commercially available robots [e.g. Webots ${ }^{\mathrm{TM}}$ robot simulator (Michel, 2004)]. The degree of realism of these robot simulations varies greatly, ranging from kinematic models of motion and collisions to the more recent physicsbased models where friction, masses, elasticity, gravity, and other physical forces are captured accurately (Bourg, 2002). Typically, the development of robotic simulations is based on systematic experiments, in which the behaviour of the physical robots and their simulated counterparts are compared until the latter prove to be reliable substitutes for the former (e.g. Mondada et al., 2004). This systematic approach ensures that the assumptions and design decisions in constructing a given model are more explicit, rigorous and less likely to be chosen for convenience.

Compared to conventional IBMs, the increase in model situatedness reduces the risk of overlooking important properties of the physical environment on social behaviour. For example, in a study on the evolution of communication using simulated robots, Mitri, Floreano \& Keller (2009) found that the clustering of foraging robots (Fig. 2B) around a food source provided inadvertent information to other robots on food location thus greatly influencing the selection pressure on communicative strategies. The resulting strategies - which depended on the existence of inadvertent information - provided an explanation for the high variability in communicative strategies in many animal species that hitherto had been difficult to explain. Models using simulated robots thus provide an advantage over conventional IBMs in scenarios where spatial and visual effects are likely to influence social behaviour.

Physics-based robotic simulations have an important advantage over the use of physical robots because they allow one to conduct numerous experiments with many individuals. This is an important issue if one wants to conduct experimental evolution over hundreds of generations. For example, an experiment on the evolution of communication where 100 groups of 10 foraging robots each had to forage for $20 \mathrm{~min}$ during each of 500 generations, lasted less than a week with a cluster of 40 computers in simulation (Mitri et al., 2009). Assuming no interruption to the experiments, the total runtime would have been almost two years for each of the three experimental treatments if the experiments were instead conducted with physical robots. Interestingly, it was possible to implement the evolved behaviour in physical robots at the end of the 500 generations of selection to confirm the robustness of the behaviour observed in the simulations (Fig. 2B).

\section{PHYSIGAL ROBOTS}

Compared to simulated robots, studies with physical robots implicitly include properties of a physical environment.
They therefore provide a particularly valuable tool when one or more of these properties are expected to be important in influencing social interactions. This is nicely illustrated by two studies on the aggregation behaviour of the German cockroach Blatella germanica. Using an IBM based on empirically measured values, Jeanson et al. (2005) attempted to reproduce the behaviour of the cockroaches. Although there was qualitative agreement between simulations and empirical data, the IBM led to larger aggregates than those formed by the cockroaches. A follow-up study with physical robots provided a better match with the empirical data (Garnier et al., 2008). An analysis of the results revealed that this was because Jeanson et al. (2005) had not included the possibility for the software agents to hide behind each other. The software agents in the IBM were thus able to perceive many more individuals in a cluster, resulting in larger and more stable aggregates than with robots and real cockroaches (Garnier et al., 2008).

Another property of robotic systems that is rarely taken into account in conventional IBMs are physical interactions between individuals that influence each other's movement. The role of such physical interactions was demonstrated in a study where a swarm of small ant-like robots was programmed to collect objects scattered in an arena (Krieger, Billeter \& Keller, 2000). These experiments revealed that foraging efficiency was lower in larger groups because there was more interference among robots than in smaller groups. This study thus provided a possible explanation for the observation that per capita productivity decreases with group size in many social insects.

Friction and body shape were found to also be crucial to collective behaviour in a study with rat-like robots aimed at mimicking how newborn rat pups form aggregations in small spaces (May et al., 2006). When setting up a baseline algorithm where robots were programmed to move randomly, the authors found that this random algorithm best fitted the collective behaviour observed in the pups. Although it was commonly thought that newborn rat pups have an instinctive attraction to objects and other rat pups (Schank et al., 2004), the study by May et al. (2006) provided a novel testable hypothesis whereby pups may simply be moving randomly and, as a result of their body shape and the friction between individuals, end up huddled in tight aggregations (see Fig. 2C, D).

\section{MIXED MODELS}

A particularly powerful application of physical robots to the study of social behaviour is the possibility of infiltrating animal societies with physical robots. Such mixed models allow the experimenter to gain insights into the animals' behavioural codes. While the use of dummies and decoys to manipulate and study animal behaviour dates back to ethologists of the 1930s and 1940s (Tinbergen, 1948), the use of robots as dummies allows the experimenter to program more sophisticated behavioural sequences and to conduct 

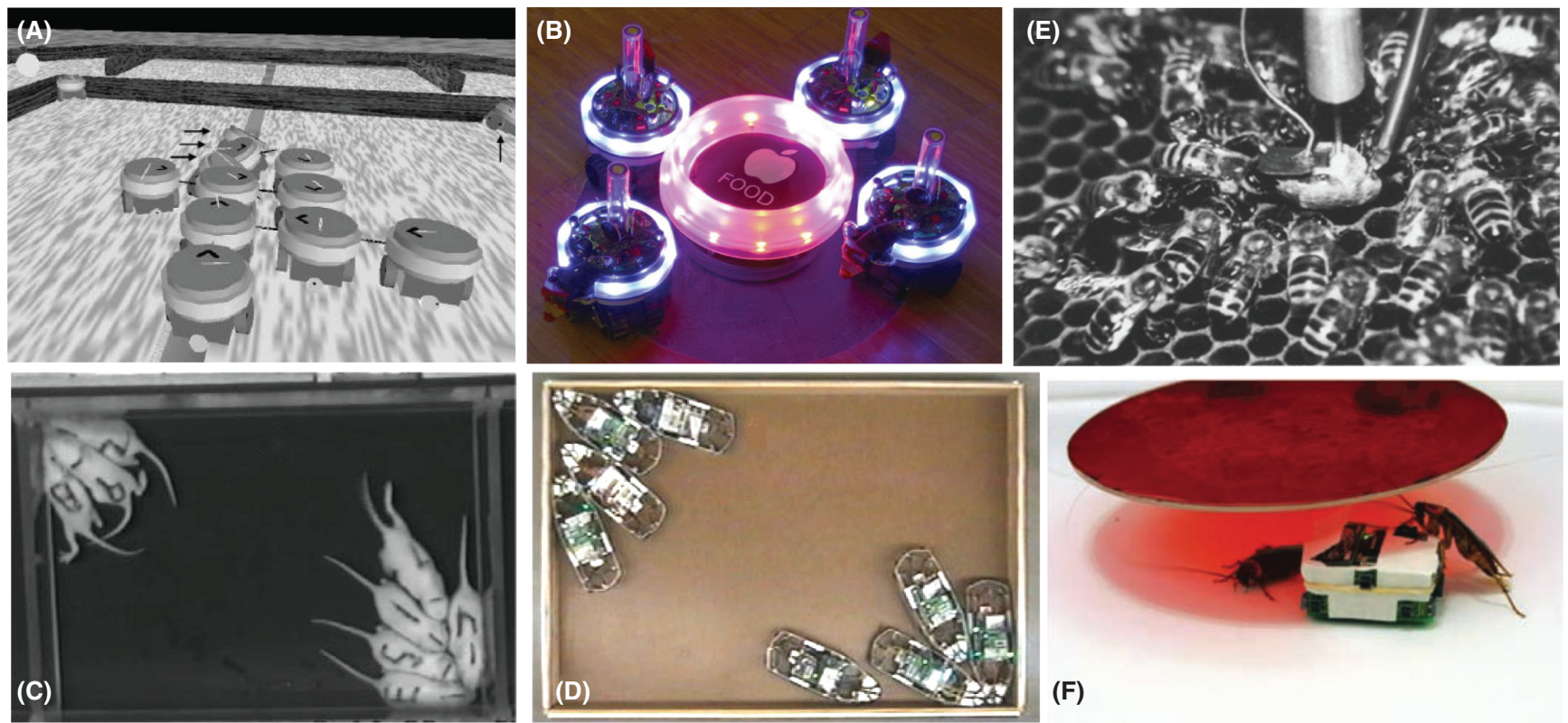

Fig. 2. Robots used to study social behaviour. (A) Simulated robots to study coordination of collective behaviour (Baldassarre, Parisi \& Nolfi, 2006). (B) Foraging robots used to study the evolution of communication (Mitri et al., 2009). (C) Modelling rat pup aggregation behaviour (G) using robots (D) (May et al., 2006). (E) Robotic bee used to test hypotheses on honeybee dance language (Michelsen et al., 1992). (F) Robots used to explore decision-making in cockroaches (Halloy et al., 2007).

closed-loop experiments where a robot can react to sensory input triggered by an animal. For example, robots were used to test how groups of cockroaches select a common shelter (Halloy et al., 2007). The decision-making process was studied by covering four robots with filter paper carrying the cockroaches' odour and mixing them with a group of 12 cockroaches. While groups of 16 cockroaches showed a preference for darker shelters, the authors found that this collective decision could be altered if the four robots were programmed to choose the less-preferred brighter shelters. From these experiments, the authors concluded that a minority of individuals could strongly influence the groups' decisions (see Fig. 2F), thus supporting conclusions drawn by previous studies using IBMs (Huse, Railsback \& Ferono, 2002; Couzin et al., 2005).

Similarly, Michelsen et al. (1992) used a robotic bee to test a long-standing controversy regarding the honeybee dance language. The use of robots permitted the authors to disentangle the roles of several components of the waggle dance on the bees' foraging behaviour, thus confirming that the waggle dance conveys abstract information on distance and direction of a source of food (Fig. 2E).

The robots used in mixed models, rather than being models in their own right, can be regarded as experimental tools used to interact with and manipulate real organisms, and therefore do not fit as cleanly into the scale of situatedness as the simulated and physical robots discussed above. Nevertheless, they have played an important and increasing role in the study of social behaviour at the intersection of robotics and biology, as reviewed by Krause, Winfield \& Deneubourg (2011). Mixed models and the use of 'cyborgs' where animal behaviour is manipulated by electronic devices have already allowed us to extend the boundaries of behavioural research and there are a number of exciting future research avenues in wildlife management, the exploration of imitation and social learning, the decoupling of morphology and behaviour and the study of social networks (Krause, Winfield \& Deneubourg, 2011).

\section{ROBOTS HAVE ADVANGED OUR UNDERSTANDING OF SOGIAL BEHAVIOUR}

The study of social behaviour has centred around understanding apparently sophisticated and complicated collective behaviour both from a mechanistic and evolutionary viewpoint. A number of important questions relating to these viewpoints have been addressed using physical robots, simulated robots or mixed models. We have compiled these into a list of 52 studies, some of which have generated novel and testable hypotheses (Table 1).

One such question is how individuals coordinate their efforts to achieve a common goal (Couzin, 2009). Experiments using both physical and simulated robots have been instrumental in showing that efficient self-organization processes can occur even with little sensory information (Holland \& Melhuish, 1999; Baldassarre, Parisi \& Nolfi, 2006; Melhuish et al., 2006; Groß et al., 2008). For example, in experiments by Groß et al. (2008), simple physical robots following identical local rules were required to retrieve a heavy 'prey' and transport it to a 'nest' - a relatively complex task that could not be conducted by any individual robot alone. 
Table 1. Articles that report on research using simulated robots, physical robots or mixed models, and explicitly state that they aim to understand social behaviour in living organisms

\begin{tabular}{|c|c|c|c|}
\hline & Simulated robots & Physical robots & Mixed models \\
\hline Hypothesis-driven & $\begin{array}{l}\text { Marocco et al. (2003), Melhuish } \\
\text { et al. (2006), Floreano et al. } \\
\text { (2007), Mitri et al. (2009), } \\
\text { Waibel et al. (2011), and } \\
\text { Wischmann } \text { et al. (2012) }\end{array}$ & $\begin{array}{l}\text { May et al. (2006), Melhuish } \\
\text { et al. (2006), Floreano et al. } \\
\text { (2007), Garnier et al. (2008), } \\
\text { Groß et al. (2008), and Garnier } \\
\text { et al. (2009) }\end{array}$ & $\begin{array}{l}\text { Michelsen } \text { et al. (1992), } \\
\text { Patricelli } \text { et al. (2002), Göth } \\
\text { \& Evans (2004), Martins } \\
\text { et al. (2005), } \\
\text { Fernández-Juricic } \text { et al. } \\
\text { (2006), Patricelli, Coleman \& } \\
\text { Borgia (2006), Halloy et al. } \\
\text { (2007), Rundus et al. (2007), } \\
\text { Ord \& Stamps (2008), } \\
\text { Reaney } \text { et al. (2008), } \\
\text { Sumpter } \text { et al. (2008), Taylor } \\
\text { et al. (2008), Partan } \text { et al. } \\
\text { (2009), Reaney (2009), and } \\
\text { Faria } \text { et al. (2010) }\end{array}$ \\
\hline Exploratory & $\begin{array}{l}\text { Mataric (1993), Di Paolo (2000), } \\
\text { Quinn (2001), Marocco et al. } \\
\text { (2003), Baldassarre et al. } \\
\text { (2006), Labella, Dorigo \& } \\
\text { Deneubourg (2006), Marocco } \\
\text { \& Nolfi (2006), and Mitri et al. } \\
\text { (2009) }\end{array}$ & $\begin{array}{l}\text { Mataric (1993), McFarland } \\
\text { (1994), Steels \& Vogt (1997), } \\
\text { Nolfi \& Floreano (1998), } \\
\text { Steels (1998), Holland \& } \\
\text { Melhuish (1999), Krieger \& } \\
\text { Billeter (2000), Krieger } \text { et al. } \\
\text { (2000), Kube \& Bonabeau } \\
\text { (2000), Vogt (2000), Birk \& } \\
\text { Wiernik (2002), Schmolke \& } \\
\text { Mallot (2002), Labella, Dorigo } \\
\text { \& Deneubourg (2006), } \\
\text { Wischmann \& Pasemann } \\
\text { (2006), Wischmann } \text { et al. } \\
\text { (2006), Garnier et al. (2007), } \\
\text { and Rubenstein } \text { et al. (2009) }\end{array}$ & $\begin{array}{l}\text { Takanishi et al. (1998), Böhlen } \\
\text { (1999), Kubinyi et al. (2004), } \\
\text { Landgraf, Moballegh \& Rojas } \\
\text { (2008), and Gribovskiy et al. } \\
\text { (2010) }\end{array}$ \\
\hline
\end{tabular}

One representative article was chosen where numerous articles reached similar conclusions. Citations in bold generated new, testable hypotheses.

The authors found that the robots self-organized into groups performing different tasks (e.g. forming a path from the object to the nest), illustrating that division of labour can take place simply due to differences in local perception, in the absence of inter-individual differences and individual recognition. Self-organization has also been shown to take place without any communication (Kube \& Bonabeau, 2000) or memory (Holland \& Melhuish, 1999). Both of these studies, in which physical robots were used, generated predictions that can be tested experimentally, for example to determine which of a number of algorithms is used by foraging ants. Other mechanisms, such as the use of simple oscillatory processes in neural networks, have been shown to lead to selforganized, synchronized light-emission patterns in groups of physical robots interacting with each other at close ranges, perhaps analogous to synchronized firefly light production (Wischmann \& Pasemann, 2006; Wischmann et al., 2006). Similarly, the production of rhythmic signals can evolve as a means to allow simulated robots to coordinate group behaviours, suggesting that turn-taking in duetting birds may serve a similar purpose (Di Paolo, 2000).

While the behaviour of robots in these studies was mostly pre-programmed, other studies have investigated how social behaviour can evolve by the mere effect of mutation and selection (Floreano \& Keller, 2010). For example, Nolfi \& Floreano (1998) have shown how competitive co-evolution between populations of predator and prey robots can drive the evolution of novel pursuit or escape strategies that were not observed when only one of the two populations was evolving. The study has also suggested that co-evolving organisms may evolve to specialize to their current opponents, a hypothesis which was later tested in co-evolving populations of bacteria and parasitic bacteriophages (Buckling \& Rainey, 2002). Experimental evolution has also been used to investigate the role of genetic relatedness on the evolution of cooperative behaviour. By allowing simulated robots to evolve in groups with different levels of relatedness, Waibel, Floreano \& Keller (2011) were able to conduct a quantitative test of Hamilton's rule (Hamilton, 1964) and demonstrate that it was possible to predict exactly the minimum relatedness required for altruism to evolve when the costs and benefits of altruistic behaviours could be controlled.

Several other studies have also focused on the evolution of communicative behaviour, a question that has been difficult to address using other experimental methods, because many forms of animal communication evolve over large 
time scales and leave no fossil record. For example, Quinn (2001) demonstrated that signals between two simulated robots can evolve from sensors that were originally used for obstacle avoidance rather than communication, thus suggesting a mechanism for the evolution of natural communication channels. Other studies have investigated how group structure may affect the likelihood to cooperate and communicate. Experimental evolution in groups of robots differing in genetic relatedness revealed that honest communication can evolve in simulated robots when communicating individuals are highly related (Floreano et al., 2007), while unrelated individuals evolve to suppress information transfer to other group members (Mitri et al., 2009; Mitri, Floreano \& Keller, 2011). Wischmann, Floreano \& Keller (2012) have similarly used experimental evolution in robot groups to explain how variations in communication systems can occur as a result of the order in which novel phenotypic traits are acquired in independently evolving populations. The study also demonstrates a trade-off between communication efficiency and robustness. Studying communicative behaviour in animal groups has also benefited from the use of robots. Mixed models have succeeded in revealing the importance of communication in some group behaviours (FernándezJuricic et al., 2006; Reaney et al., 2008), disentangling the different components of signalling systems (Michelsen et al., 1992; Patricelli et al., 2002; Göth \& Evans, 2004; Martins, Ord \& Davenport, 2005; Rundus et al., 2007), and showing how these components interact to increase communication efficiency in noisy environments (Ord \& Stamps, 2008; Taylor et al., 2008; Partan, Larco \& Owens, 2009).

Wheeled and humanoid robots have similarly been used to investigate human language and explore how continuous perceptual data can be mapped onto discrete 'words' or symbols (Harnad, 1990). These studies have established that interacting with the environment and with other simulated or physical robots can lead to categorization of perceptual data (Marocco, Cangelosi \& Nolfi, 2003; Marocco \& Nolfi, 2006), the formation of 'vocabularies' (Steels \& Vogt, 1997; Vogt, 2000) and even simple forms of syntax (Steels, 1998). Because the perceptual data that a robot is exposed to, as opposed to a simulated individual, is similar in richness to information collected by a living organism, the use of robots has proven to be a valuable tool in understanding how living organisms categorize information and map it onto symbols. For example, a robot may approach the same object from different sides, but will still need to recognize the object in order to assign a name to it (Loetzsch \& Spranger, 2010).

\section{EVALUATING ROBOTIG MODELS}

Although robotic models have been useful in addressing a variety of issues on social behaviour, these studies are only infrequently integrated into the biological literature. Subsequently, hardly any of the hypotheses generated by robotic models outlined above have been tested using living organisms. There are at least two main reasons for this. The first is that many of these studies are published in computer science or artificial life journals that are infrequently read by the biological community. The second reason is that many of these studies do not use a 'hypothesis-driven' approach [Table 1, see also 'strong inference' (Platt, 1964)]. Currently, much of the work in biology consists of hypothesis-driven experiments with researchers designing experiments to test a specific hypothesis. So far only a minority of the experiments conducted with robotic models are of this type. Most have been 'exploratory' with a model being constructed to explore whether the implicit inclusion of the robots' physical properties will reveal novel aspects concerning the collective behaviour in question. While such exploratory studies may be less constrained in how they approach a given problem, and thus may uncover unexpected patterns, they carry the risk of not answering a particular question. Furthermore, because these studies are not designed to test a specific hypothesis, the results are usually less convincing and it may be more difficult for a biological audience to draw parallels and apply these findings to their own work. In hypothesis-driven experiments, on the other hand, controlled manipulations make it easier to understand causal mechanisms, and thus to link the model results to similar natural phenomena.

Since the birth of the scientific method, scientists and philosophers have been debating the most promising and efficient methods to improve our understanding of nature. In the 17th Century, Francis Bacon discussed this point, asking whether researchers should collect data in an exploratory manner 'without premature reflection or any great subtlety' (Bacon, 1620). These observations could then be used to construct scientific theories. In a recent article, Franklin (2005) argued that scientists tend to resort to such exploratory methods when a new tool becomes available for data collection, and when that tool allows for the collection of vast amounts of data. This allows them to map out new scientific territory. Consistent with Franklin's (2005) analysis, researchers studying social behaviour with robots have initially mostly conducted exploratory studies to determine whether the embodiment of agents in a physical world leads to surprising outcomes increasing our understanding of social behaviour. More recently, the trend appears to have shifted towards a higher proportion of hypothesis-driven studies (see Table 1 and Fig. 3).

The shift from exploratory to hypothesis-driven experiments is to a large extent due to a recent increase in studies using mixed models. This is perhaps because mixed models are often designed by researchers working on a living system and who use robots to address a specific question that could not be answered otherwise. The robots thus often serve as an experimental tool to vary parameters systematically and test for their effects. These hypothesis-driven studies also appear to have led to a greater number of novel hypotheses that can be tested in a biological context than exploratory studies (see highlighted studies in Table 1).

Another reason why biologists might be reluctant to utilize results from robotic studies is that it is not always clear how the behavioural code of the robot is implemented and 


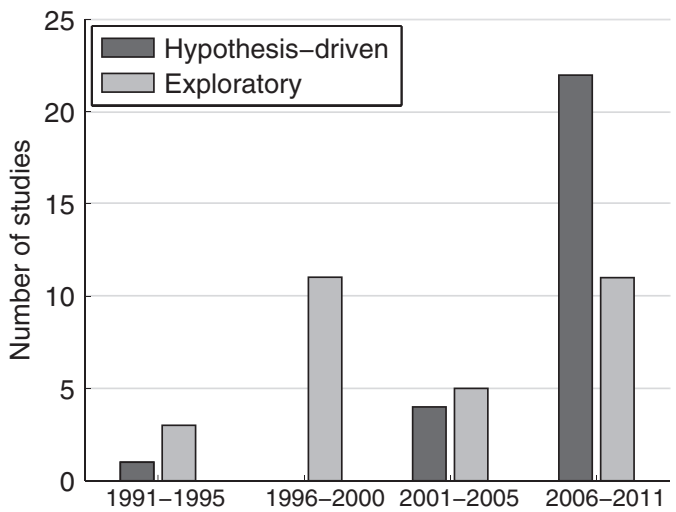

Fig. 3. Histogram of the number of hypothesis-driven versus exploratory studies over the last two decades.

whether this implementation is biologically relevant. One way to overcome this problem would be for modellers to define explicitly and objectively the behavioural code that is used by a robot in order to provide the reader with sufficient information to evaluate the results of a study and its parallels in biological organisms.

\section{SUMMARY AND FUTURE DIRECTIONS}

Our review of the literature shows that robots have contributed to our understanding of social behaviour, both through exploratory studies, whereby the complexity of the robots' social behaviour in a physical environment is used to inspire further empirical studies, and through hypothesisdriven experiments. Robots represent a modelling niche by occupying the situatedness space between agent-based models and real organisms. Because one has full access to the sensors and actuators of a robot, it is easier to control and understand than a living organism. At the same time, the increase in situatedness compared to agent-based computer simulations provides an advantage whenever certain physical features of the real world are expected to influence social behaviour or when the question is specifically about the influence of certain physical properties that can be considered with the robot. The examples listed in this review illustrate how visual and spatial effects can influence aggregation, search or communicative behaviours (Jeanson et al., 2005; Garnier et al., 2008; Mitri et al., 2009), and how friction and collisions between individuals can play an important role in collective foraging (Krieger \& Billeter, 2000; Krieger et al., 2000; Kube \& Bonabeau, 2000; Waibel et al., 2011) and clustering behaviours (Holland \& Melhuish, 1999; May et al., 2006; Melhuish et al., 2006).

However, the benefit of using robotic models rather than conventional IBMs depends on the particular question one is addressing. Ideally, the chosen model will be similar enough to the real system to include all the factors of interest and relevance, yet simple enough to allow control over the various parameters of the model and adequate analysis of the results.
Consequently, robots are not always the tool of choice. Rather, they should be considered only when the properties of a robot, physical or simulated, are likely to influence the outcome of a particular experiment. Furthermore, if the conclusions of a study using robots are found to be independent of any such physical properties, one can then reduce the complexity of the study to a simpler model that captures the natural observation more concisely. A further limitation of using robots is that some observations may be due to artefacts that are particular to the robots, and may not correspond to any natural phenomena. This problem can be avoided by conducting a detailed analysis of the mechanisms responsible for an observed behaviour, and making concrete comparisons with empirical biological data (Webb, 2009).

Nevertheless, we believe that there is much potential for the use of robots that has yet to be explored. For example, there is much discussion on how individual and kin recognition affect decisions made within a collective (Sheehan \& Tibbetts, 2008; Paterson \& Hurst, 2009; Nehring et al., 2011). Because such recognition is based on noisy sensory information, distinguishing between individuals may be difficult both for animals and robots that must solve the task in the physical world. It would be interesting to see whether similarities in sensory perception between animals and robots - compared to abstract agents - may give some insights into the role of peer recognition in collective decision-making. In robots and animals one might, for example, expect a certain level of error in recognizing others that may influence the social dynamics of the group.

Another potential use of robots is to study the interplay between mechanistic properties that are highly dependent on physical factors, and effects arising over evolutionary time. Mayr (1961) and Tinbergen (1963) were perhaps the first to argue for the synergistic benefits of a complementary approach addressing both proximate (mechanistic) and ultimate (evolutionary) causes. As has been discussed herein, the use of evolutionary robotics has been instrumental in exploring such questions in groups of robots, and has resulted in important contributions to our understanding of the evolution of social behaviour, thus highlighting the strength of robotic models. Such a complementary approach could be similarly applied with mixed models to study the interplay between behavioural and evolutionary processes. A nice illustration of this idea is provided by a study where blue jays (Cyanocitta cristata) searched for digital moths on computer monitors (Bond \& Kamil, 2002). The digital moths that were not pecked by the birds survived to subsequent generations, thus allowing to investigate how selection shapes phenotypic properties of moths. Although this study did not involve robots, it may inspire similar studies using mixed models where the robots' controllers or morphology can evolve in parallel with organisms with a short generation time. Such an approach provides a unique opportunity to investigate how social interactions can affect the evolutionary pathway of organisms and the evolution of complex social systems (D'Eath, 1998; Rosenthal \& Evans, 1998; Baldauf, Kullmann \& Bakker, 2008; Moiseff \& Copeland, 2010). 
Finally, it is important to note that physical robots have become cheaper and simulations easier to design and perform. Robotic research platforms have also become available, such as the Symbrion system, which consists of hundreds of miniature robots that can be used for evolutionary and collective experiments (Kernbach et al., 2008). This should contribute to the more frequent use of robotic systems to address biological questions that are difficult or impossible to address with real organisms.

\section{GONGLUSIONS}

(1) Robots have made important contributions to the study of self-organization, communication and the evolution of competitive and cooperative behaviour.

(2) Both exploratory and hypothesis-driven studies using robots have generated novel hypotheses that can be tested using living organisms. However, the more recent shift towards hypothesis-driven studies is likely to make these findings more accessible to the biological community.

(3) The choice of modelling tool depends on the question of interest and can be selected from different levels of 'situatedness'. The model should be similar enough to the real system being studied to include all components that are relevant to generating the observed empirical data, yet simple enough to be amenable to detailed analysis and understanding.

(4) Robots are useful when properties of the physical environment (e.g. visual and spatial effects, friction and collisions) are likely to influence the outcome of the social behaviour.

\section{AGKNOWLEDGEMENTS}

We thank Michel Chapuisat, Volker Grimm, José Halloy, Brian Hollis, Eric Lucas and two anonymous reviewers for helpful comments on the manuscript. This research has been supported by the Swarmanoid project founded by the Future and Emerging Technologies program (IST-FET) of the European Community under EU R\&D contract IST022888, by an ERG advanced grant and by the Swiss National Science Foundation.

\section{REFERENGES}

Bacon, F. (1620). The New Organon. Cambridge University Press, Cambridge. Baldassarre, G., Parisi, D. \& Nolfi, S. (2006). Distributed coordination of simulated robots based on self-organization. Artificial Life 12, 289-311.

Baldauf, S. A., Kullmann, H. \& Bakker, T. C. M. (2008). Technical restrictions of computer-manipulated visual stimuli and display units for studying animal behaviour. Ethology 114, 737-751.

Birk, A. \& Wiernik, J. (2002). An N-player prisoner's dilemma in a robotic ecosystem. Robotics and Autonomous Systems 39, 223-233.

BöHLEN, M. (1999). A robot in a cage-exploring interactions between animals and robots. IEEE International Symposium on Computational Intelligence in Robotics and Automation (Volume 12), pp. 214-219. IEEE Press, Monterey.
Bond, A. B. \& Kamil, A. C. (2002). Visual predators select for crypticity and polymorphism in virtual prey. Nature 415, 609-613.

Bourg, D. M. (2002). Physics for Game Developers. O'Reilly \& Associates Inc., Sebastopol, CA, USA.

Buckling, A. \& Rainey, P. B. (2002). Antagonistic coevolution between a bacterium and a bacteriophage. Proceedings of the Roval Society London B 269, 931-936.

Clark, A. (1996). Being There: Putting Brain, Body, and World Together Again. MIT Press, Cambridge.

Couzin, I. D. (2009). Collective cognition in animal groups. Trends in Cognitive Sciences 13, 36-43.

Couzin, I. D., Krause, J., Franks, N. R. \& Levin, S. A. (2005). Effective leadership and decision-making in animal groups on the move. Nature 433, 513-516.

DeAngelis, D. L. \& Mooij, W. M. (2005). Individual-based modeling of ecological and evolutionary processes. Annual Review of Ecology, Evolution, and Systematics 36, $147-168$.

D'EAтH, R. B. (1998). Can video images imitate real stimuli in animal behaviour experiments? Biological Reviewes of the Cambridge Philosophical Society 73, 267-292.

Di Paolo, E. A. (2000). Behavioral coordination, structural congruence and entrainment in a simulation of acoustically coupled agents. Adaptive Behavior 8, $25-46$.

faria, J., Dyer, J. R. G., Clément, R. O., Couzin, I. D., Holt, N., Ward, A. J. W., Waters, D. \& Krause, J. (2010). A novel method for investigating the collective behaviour of fish: introducing 'Robofish'. Behavioral Ecology and Sociobiology 64, 1211-1218.

Fernández-Juricic, E., Gilak, N., McDonald, J., Pithia, P. \& Valcarcel, A. (2006). A dynamic method to study the transmission of social foraging information in flocks using robots. Animal Behaviour 71, 901-911.

Floreano, D. \& Keller, L. (2010). Evolution of adaptive behaviour in robots by means of Darwinian selection. PLoS Biology 8, e1000292.

Floreano, D., Mitri, S., Magnenat, S. \& Keller, L. (2007). Evolutionary conditions for the emergence of communication in robots. Current Biology 17, $514-519$.

Franklin, L. R. (2005). Exploratory experiments. Philosophy of Science 72, 888-899.

Garnier, S. (2011). From ants to robots and back: how robotics can contribute to the study of collective animal behavior. Bio-Inspired Self-Organizing Robotic Systems, pp. 105-120. Springer-Verlag, Berlin.

Garnier, S., Gautrais, J., Asadpour, M., Jost, C. \& Theraulaz, G. (2009). Self-organized aggregation triggers collective decision making in a group of cockroach-like robots. Adaptive Behavior 17, 109-133.

Garnier, S., Jost, C., Gautrais, J., Asadpour, M., Caprari, G., Jeanson, R., Grimal, A. \& Theraulaz, G. (2008). The embodiment of cockroach aggregation behavior in a group of micro-robots. Artificial Life 14, 387-408.

Garnier, S., TÂche, F., Combe, M., Grimal, A. \& Theraulaz, G. (2007). Alice in pheromone land: an experimental setup for the study of ant-like robots. In Proceedings of the 2007 IEEE Swarm Intelligence Symposium (SIS 2007), pp. 37-44. Honolulu, Hawaii.

GöTH, A. \& Evans, C. S. (2004). Social responses without early experience: Australian brushturkey chicks use specific visual cues to aggregate with conspecifics. Fournal of Experimental Biology 207, 2199-2208.

Gribovskiy, A., Halloy, J., Deneubourg, J.-L., Bleuler, H. \& Mondada, F. (2010). Towards mixed societies of chickens and robots. In 2010 IEEE/RSf International Conference on Intelligent Robots and Systems, pp. 4722-4728. Taipei, Taiwan.

Grimm, V. \& Railsback, S. F. (2005). Individual-Based Modeling and Ecology. Princeton University Press, Princeton.

Gross, R., Nouyan, S., Bonani, M., Mondada, F. \& Dorigo, M. (2008). Division of Labour In Self-Organised Groups. Lectures in Computer Science, from Animals to Animats 10, Vol. 5040. Springer, Berlin.

Halloy, J., Sempo, G., Caprari, G., Rivault, C., Asadpour, M., Tâche, F., Saï̀, I., Durier, V., Canonge, S., Amé, J. M., Detrain, C., Correll, N., Martinoli, A., Mondada, F., Siegwart, R. \& Deneubourg, J.-L. (2007). Social integration of robots into groups of cockroaches to control self-organized choices. Science 318, 1155-1158.

Hamilton, W. D. (1964). The genetical evolution of social behaviour. Fournal of Theoretical Biology 7, 1-52.

Harnad, S. (1990). The symbol grounding problem. Physica D 42, 335-346.

Holland, O. \& Melhuish, C. (1999). Stigmergy, self-organization, and sorting in collective robotics. Artificial Life 5, 173-202.

Huse, G., Railsback, S. \& Ferono, A. (2002). Modelling changes in migration pattern of herring: collective behaviour and numerical domination. Fournal of Fish Biology 60, 571-582.

Jeanson, R., Rivault, G., Deneubourg, J.-L., Blanco, S., Fournier, R., Jost, C. \& Theraulaz, G. (2005). Selforganized aggregation in cockroaches. Animal Behaviour 69, 169-180.

Judson, O. P. (1994). The rise of the individual-based model in ecology. Trends in Ecology \& Evolution 9, 9-14.

Kernbach, S., Szymanski, M., Schmickl, T., Corradi, P. \& Anna, S. (2008). Symbiotic robot organisms: replicator and Symbrion projects. In Proceedings of 
Performance Metrics for Intelligent Systems Workshop (PerMIS 08), pp. 62-69. Washington, DC, USA.

Krause, J., Winfield, A. F. T. \& Deneubourg, J.-L. (2011). Interactive robots in experimental biology. Trends in Ecology \& Evolution 26, 369-375.

Krieger, M. J. B. \& Billeter, J.-B. (2000). The call of duty: self-organised task allocation in a population of up to twelve mobile robots. Robotics and Autonomous Systems 30, 65-84.

Krieger, M. J. B., Billeter, J.-B. \& Keller, L. (2000). Ant-like task allocation and recruitment in cooperative robots. Nature 406, 992-995.

Kube, C. R. \& Bonabeau, E. (2000). Cooperative transport by ants and robots. Robotics and Autonomous Systems 30, 85-101.

Kubinyi, E., Miklósi, Á., Kaplan, F., Gácsi, M., Topál, J. \& Csányi, V. (2004). Social behaviour of dogs encountering AIBO, an animal-like robot in a neutral and in a feeding situation. Behavioural Processes 65, 231-239.

Labella, T. H., Dorigo, M. \& Deneubourg, J.-L. (2006). Division of labor in a group of robots inspired by ants' foraging behavior. ACM Transactions on Autonomous and Adaptive Systems 1, 4-25.

Landgraf, T., Moballegh, H. \& Rojas, R. (2008). Design and development of a robotic bee for the analysis of honeybee dance communication. Applied Bionics and Biomechanics 5, 157-164.

Loetzsch, M. \& Spranger, M. (2010). Why robots? In The Evolution of Language (Evolang8) (eds A. Smith, M. Schouwstra, B. DE Boer and K. SMith), pp. 222-229. World Scientific, Singapore.

Marocco, D., Cangelosi, A. \& Nolfi, S. (2003). The emergence of communication in evolutionary robots. Philosophical Transactions of the Royal Society London A 361, 2397-2421.

Marocco, D. \& Nolfi, S. (2006). Self-organization of communication in evolving robots. In Proceedings of the Tenth International Conference on Artificial Life (eds L. M. Rocha， L. Yaeger， M. Bedau， D. Floreano， R. Goldstone and A. Vespignani), pp. 199-205. MIT Press, Cambridge.

Martins, E. P., Ord, T. J. \& Davenport, S. W. (2005). Combining motions into complex displays: playbacks with a robotic lizard. Behavioral Ecology and Sociobiology 58, $351-360$.

Mataric, M. J. (1993). Kin recognition, similarity, and group behavior. Proceedings of the Fifteenth Annual Conference of the Cognitive Science Society, pp. 705-710. Lawrence Erlbaum Associates, Philadelphia.

May, C. J., Schank, J. G., Joshi, S. S., Tran, J. T., Taylor, R. J. \& Scott, I.-E. (2006). Rat pups and random robots generate similar self-organized and intentional behavior. Complexity 12, 53-66.

MAYR, E. (1961). Cause and effect in biology: kinds of causes, predictability, and teleology are viewed by a practicing biologist. Science 134, 1501-1506.

McFarland, D. (1994). Towards robot cooperation. In From Animals to Animats 3: Proceedings of the Third International Conference on Simulation of Adaptive Behavior, pp. 440-444. Brighton, UK.

Melhuish, C., Sendova-Franks, A. B., Scholes, S., Horsfield, I. \& Welsby, F. (2006). Ant-inspired sorting by robots: the importance of initial clustering. Fournal of the Royal Society Interface 3, 235-242.

MicheL, O. (2004). Webots: professional mobile robot simulation. International foumal of Advanced Robotic Systems 1, 39-42.

Michelsen, A., Andersen, B. B., Storm, J., Kirchner, W. H. \& Lindauer, M. (1992). How honeybees perceive communication dances, studied by means of a mechanical model. Behavioral Ecology and Sociobiology 30, 143-150.

Mitri, S., Floreano, D. \& Keller, L. (2009). The evolution of information suppression in communicating robots with conflicting interests. Proceedings of the National Academy of Sciences of the United States of America 106, 15786-15790.

Mitri, S., Floreano, D. \& Keller, L. (2011). Relatedness influences signal reliability in evolving robots. Proceedings of the Royal Society London B 278, 378-383.

Moiseff, A. \& Copeland, J. (2010). Firefly synchrony: a behavioral strategy to minimize visual clutter. Science $329,181$.

Mondada, F., Pettinaro, G. C., Guignard, A., Kwee, I., Floreano, D., Deneubourg, J.-L., Nolfi, S., Gambardella, L. M. \& Dorigo, M. (2004). Swarm-Bot: a new distributed robotic concept. Autonomous Robots 17, 193-221.

Nehring, V., Evison, S. E. F., Santorelli, L. A., D’Ettorre, P. \& Hughes, W. O. H. (2011). Kin-informative recognition cues in ants. Proceedings of the Royal Society London B 278, 1942-1948.

Nolfi, S. \& Floreano, D. (1998). Co-evolving predator and prey robots: do "arm races" arise in artificial evolution. Artificial Life 4, 311-335.

Ord, T. J. \& STAMPS, J. A. (2008). Alert signals enhance animal communication in "noisy" environments. Proceedings of the National Academy of Sciences of the United States of America 105, 18830-18835.

Partan, S. R., Larco, C. P. \& Owens, M. J. (2009). Wild tree squirrels respond with multi-sensory enhancement to conspecific robot alarm behaviour. Animal Behaviour $77,1127-1135$.
Paterson, S. \& Hurst, J. L. (2009). How effective is recognition of siblings on the basis of genotype? Fournal of Evolutionary Biology 22, 1875-1881.

Patricelli, G., Coleman, S. \& Borgia, G. (2006). Male satin bowerbirds, Ptilonorhynchus violaceus, adjust their display intensity in response to female startling: an experiment with robotic females. Animal Behaviour 71, 49-59.

Patricelli, G. L., Uy, J. A. C., Walsh, G. \& Borgia, G. (2002). Male displays adjusted to female's response. Nature 415, 279-280.

Platt, J. (1964). Strong inference. Science 14, 347-353.

QuinN, M. (2001). Evolving communication without dedicated communication channels. In Advances in Artificial Life: The Sixth European Conference (eds J. KeLEMEN and P. Solvík), pp. 357-366. Springer, Berlin.

Reaney, L. T. (2009). Female preference for male phenotypic traits in a fiddler crab: do females use absolute or comparative evaluation? Animal Behaviour 77, 139-143.

Reaney, L. T., Sims, R. A., Sims, S. W. M.,Jennions, M. D. \& Backwell, P. R. Y. (2008). Experiments with robots explain synchronized courtship in fiddler crabs. Current Biology 18, R62-R63.

Rosenthal, G. G. \& Evans, C. S. (1998). Female preference for swords in Xiphophorus helleri reflects a bias for large apparent size. Proceedings of the National Academy of Sciences of the United States of America 95, 4431-4436.

Rubenstein, M., SaI, Y., Chuong, C.-M. \& Shen, W.-M. (2009). Regenerative patterning in swarm robots: mutual benefits of research in robotics and stem cell biology. The International fournal of Developmental Biology 53, 869-881.

Rundus, A. S., Owings, D. H., Joshi, S. S., Chinn, E. \& Giannini, N. (2007). Ground squirrels use an infrared signal to deter rattlesnake predation. Proceedings of the National Academy of Sciences of the United States of America 104, 14372-14376.

Schank, J. C., May, C. J., Tran, J. T. \& Joshi, S. S. (2004). A biorobotic investigation of Norway rat pups (Rattus norvegicus) in an arena. Adaptive Behavior $12,161-173$.

Schmolke, A. \& Mallot, H. A. (2002). Territory formation in mobile robots. In Artificial Life VIII (eds R. Standish, H. AbBass and M. BeDau), pp. 256-259. MIT Press, Cambridge.

Sheehan, M. J. \& Tibbetts, E. A. (2008). Robust long-term social memories in a paper wasp. Current Biology 18, R851-R852.

STEELS, L. (1998). The origins of syntax in visually grounded robotic agents. Artificial Intellisence 103, 133-156.

Steels, L. \& Vogt, P. (1997). Grounding adaptive language games in robotic agents. In Fourth European Conference on Artificial Life (eds P. Husbands and P. HarveY), pp. 474-482. MIT Press, Cambridge.

Sumpter, D. J. T., Krause, J., James, R., Couzin, I. D. \& Ward, A. J. W. (2008). Consensus decision making by fish. Current Biology 18, 1773-1777.

Takanishi, A., Aoki, T., Ito, M., Ohkawa, Y. \& Yamaguchi, J. (1998). Interaction between creature and robot: development of an experiment system for rat and rat robot interaction. In IEEE/RSF International Conference on Intelligent Robots and Systems, pp. 1975-1980. Victoria, BC, Canada.

TAYlor, R. C., Klein, B. A., Stein, J. \& Ryan, M. J. (2008). Faux frogs: multimodal signalling and the value of robotics in animal behaviour. Animal Behaviour 76, $1089-1097$.

Tinbergen, N. (1948). Social releasers and the experimental method required for their study. The Wilson Bulletin 60, 6-51.

Tinbergen, N. (1963). On aims and methods of ethology. Zeitschrift fur Tierpsychologie $20,410-433$.

Varela, F., Thompson, E. \& Rosch, E. (1991). The Embodied Mind: Cognitive Science and Human Experience. MIT Press, Cambridge.

Vogt, P. (2000). Lexicon grounding on mobile robots. Unpublished Doctoral Dissertation, Vrije Universiteit Brussel.

Waibel, M., Floreano, D. \& Keller, L. (2011). A quantitative test of Hamilton's rule for the evolution of altruism. PLoS Biology 9, e1000615.

WALDNER, J.-B. (2008). Nanocomputers and Swarm Intelligence. Wiley-ISTE, London.

Wевв, B. (2009). Animals versus animats: or why not model the real iguana? Adaptive Behavior 17, 269-286.

Wischmann, S., Floreano, D. \& Keller, L. (2012). Historical contingency affects signaling strategies and competitive abilities in evolving populations of simulated robots. Proceedings of the National Academy of Sciences of the United States of America 109, 864-868.

Wischmann, S., Hülse, M., Knabe, J. F. \& Pasemann, F. (2006). Synchronization of internal neural rhythms in multi-robot systems. Adaptive Behavior 14, 117-127.

Wischmann, S. \& Pasemann, F. (2006). The emergence of communication by evolving dynamical systems. In From Animals to Animats 9: 9th International Conference on Simulation of Adaptive Behavior, SAB 2006 (eds S. Nolfi, G. Baldassarre, R. Calabretta, J. C. T. Hallam, D. Marocco, J. A. Meyer, O. Miglino and D. PARISI), pp. 777-788. Springer-Verlag, Berlin.

(Received 21 December 2011; revised 29 May 2012; accepted 15 Fune 2012; published online 21 Fuly 2012) 